\title{
Development of risk factor-based scoring system for detection of hypervirulent Klebsiella pneumoniae bloodstream infections
}

\author{
Atsushi Togawa ${ }^{1,2^{*}}$ (D), Michinobu Yoshimura ${ }^{1,4 \dagger}$, Chiemi Tokushige ${ }^{3 \dagger}$, Akira Matsunaga $^{3}$, Tohru Takata ${ }^{1,2}$ \\ and Yasushi Takamatsu'
}

\begin{abstract}
Background: Hypervirulent Klebsiella pneumoniae (HVKp) infections have distinct clinical manifestations from classical K. pneumoniae infections. The hallmark of HVKp infections are liver abscess formation and metastatic infections. Due to the severe sequelae of these complications, method to identify patients at-risk of HVKp infections should be developed.

Results: A retrospective cohort study of 222 patients with K. pneumoniae bloodstream infections (BSIs) was performed. Patient demographics, clinical manifestations, and bacterial characteristics were investigated. Ten cases of liver abscesses were identified. Characteristics such as community-onset BSIs, hypermucoviscosity phenotype, and capsular serotype K1 were identified as risk factors for HVKp infections. A scoring system was developed based on the risk factors. The area under the receiver operating characteristic curve for the scoring system was 0.90 . A score of $\geq 2$ points provided sensitivity and specificity of 0.70 and 0.94 , respectively.

Conclusions: Simple scoring system was developed for the diagnosis of HVKp infections. The system allows early identification of patients with K. pneumoniae BSIs in whom hypervirulent infections should be evaluated. Prospective evaluation is expected.
\end{abstract}

Keywords: Hypervirulent Klebsiella pneumoniae, Bloodstream infection, Hypermucoviscosity, Capsular serotype, Scoring system

\section{Background}

Klebsiella pneumoniae is a common hospital-acquired pathogen, causing classical K. pneumoniae (CKp) infections such as pneumonia, urinary tract infection, intra-abdominal infection and bacteremia in immunocompromised patients [1]. In 1986, physicians from Taiwan reported seven cases of pyogenic liver abscess

\footnotetext{
*Correspondence: atogawa@fukuoka-u.ac.jp

${ }^{\dagger}$ Michinobu Yoshimura and Chiemi Tokushige contributed equally to this work

${ }^{1}$ Department of Medical Oncology, Hematology, and Infectious Diseases, Fukuoka University Hospital, 7-45-1 Nanakuma, Jonan, Fukuoka 8140180, Japan

Full list of author information is available at the end of the article
}

associated with septic endophthalmitis by $K$. pneumoniae [2]. A similar case was reported from Spain later [3]. Since then the number of reported cases of pyogenic liver abscesses and associated metastatic infections, such as endophthalmitis, meningitis, and necrotizing fasciitis, by $K$. pneumoniae is rising [4-6]. These symptoms have never been observed in CKp infections.

The clinical and bacterial characteristics of these invasive syndromes, named as hypervirulent $K$. pneumoniae (HVKp) infections, have been extensively studied [6-8]. The distinctive characters of HVKp infections could be summarized as follows; (1) they are predominantly reported from Taiwan and Southeast Asia compared to the worldwide distribution of CKp infections; (2)

c) The Author(s) 2020. This article is licensed under a Creative Commons Attribution 4.0 International License, which permits use, sharing, adaptation, distribution and reproduction in any medium or format, as long as you give appropriate credit to the original author(s) and the source, provide a link to the Creative Commons licence, and indicate if changes were made. The images or other third party material in this article are included in the article's Creative Commons licence, unless indicated otherwise in a credit line to the material. If material is not included in the article's Creative Commons licence and your intended use is not permitted by statutory regulation or exceeds the permitted use, you will need to obtain permission directly from the copyright holder. To view a copy of this licence, visit http://creativecommons.org/licenses/by/4.0/. The Creative Commons Public Domain Dedication waiver (http://creativecommons.org/publicdomain/zero/1.0/) applies to the data made available in this article, unless otherwise stated in a credit line to the data. 
common types of HVKp infections almost always include pyogenic liver abscess and, occasionally, other metastatic infections; (3) the typical mode of HVKp infections is community-onset infection in both immunocompetent and immunocompromised patients, compared to CKp which usually causes nosocomial infection in immunocompromised patients; (4) strains isolated from HVKp infections possess the hypermucoviscosity phenotype, which reflects the hyperproduction of capsules and can easily be detected by the string test [9], and the preferential distribution of capsular serotypes K1 and K2 [10].

Risk factors for HVKp infections in patients with $K$. pneumoniae bloodstream infections (BSIs) has been studied. Li et al. showed that diabetes mellitus and community-acquired BSIs are independent risk factors for HVKp BSIs [11]. Harada et al. reported that patients with HVKp infection had higher proportions of diabetes mellitus, and their infections had significantly higher propensity to liver abscess formation among Japanese patients with K. pneumoniae BSIs [12]. In these studies, the definitions of HVKp infections were based on molecular assays, in which the detection of virulence-associated genes, such as $\operatorname{rmp} A$, iroBCDN, iucABCD, and iutA, was a prerequisite for defining them as HVKp.

Even though the diagnostic criteria of HVKp infections has still not been formally established [7], definition of HVKp infections should be based on clinical manifestations rather than microbiological analyses, since molecular analyses of virulence genes do not seems to be feasible in many microbiology laboratories. For precise surveillance of the incidence and clinical spectrum of HVKp infections, proper diagnostic criteria should be established, and if microbiology methods were to be applied, they should be as simple as possible to be used as routine practice in microbiology laboratories. In addition, based on such criteria, methods to identify patients with K. pneumoniae BSIs who are at-risk of HVKp infections should be investigated.

In this study we enrolled patients with $K$. pneumoniae BSIs for 10-year period and analyzed the clinical and bacterial characteristics to clarify the risk factors for HVKp infections. Based on the identified risk factors we tried to develop a scoring system to identify patients with $K$. pneumoniae BSIs who are at-risk of HVKp infections.

\section{Results}

222 unique episodes of $K$. pneumoniae BSIs were identified, of which 103 (46\%) episodes were community-onset. Since community-onset infection is one of the hallmarks of HVKp infections, we compared clinical manifestations of community-onset BSIs with those of hospitalonset BSIs (Table 1). Comparing to hospital-onset BSIs, patients with community-onset BSIs were older (72.6 v.
64.4) and biliary tract diseases were more often observed as underlying disease $(34.0 \%$ v. $14.3 \%)$. Five patients (4.9\%) with community-onset BSIs had no underlying disease, whereas all patients with hospital-onset BSIs had underlying diseases. Most notably, liver abscess was observed only in patients with community-onset BSIs. No significant difference was observed in antibacterial treatments in both patient cohorts, and the 30-day survival was comparable ( $82.3 \%$ v. $84.6 \%)$.

Analyses of isolated strains from these $K$. pneumoniae infections revealed several distinct characters with community-onset BSIs. Hypermucoviscosity phenotype was more often observed in community-onset BSIs than in hospital-onset BSIs (14.6\% v. 5.0\%). When the expression of $r m p A$ gene, which is associated with the production of capsules [13], was determined in 133 strains, the gene was preferentially expressed in strains isolated from patients with community-onset BSIs than with hospital-onset BSIs (21.2\% v. 6.2\%). In terms of antimicrobial resistance, ESBL-producing $K$. pneumoniae was detected in $1.9 \%$ and $18.5 \%$ of patients with community-onset and hospital-onset BSIs, respectively. Resistance to carbapenems was observed in only one case of hospital-onset BSI.

Since liver abscess formation is uniformly associated with HV $K$. pneumoniae infections, we checked the medical records to identify patients in whom abdominal computed tomography (CT) scans were performed. We looked for cases with abdominal CT scans available and excluded cases examined with abdominal echograms, since abdominal CT scan is the most reliable method to detect liver abscesses caused by $K$. pneumoniae $[14,15]$. Among our patient cohort, 127 (57.2\%) patients received abdominal CT scans irrespective of contrast-enhancement within 7 days after the onset of $K$. pneumoniae BSIs. Abdominal CT scans were preferentially performed in patients aged 65 or older (odds ratio (OR), 1.98; 95\% confidence interval (CI) 1.12-3.51) or in cases of community-onset BSIs (OR, 2.70; 95\% CI 1.55-4.70) by univariate analyses.

We found 10 cases of liver abscesses in patients checked by abdominal CT scans. Among these cases we found a case complicated by endophthalmitis and another case with vertebral osteomyelitis and epidural abscess. In the former case, onset of K. pneumoniae BSI and the endophthalmitis was at the same time. However, in the second case, lower back pain has developed after the onset of $K$. pneumoniae BSI, and the vertebral osteomyelitis and epidural abscess was diagnosed after 3 weeks from the onset of BSI by magnetic resonance imaging.

When cases with and without liver abscesses were compared, several differences in clinical manifestations and bacterial characteristics were found (Table 2). All cases of liver abscesses were observed in community-onset BSIs, 
Table 1 Clinical and bacterial characteristics of $K$. pneumoniae BSIs

\begin{tabular}{|c|c|c|c|c|c|}
\hline \multirow[t]{2}{*}{ Characteristics } & \multicolumn{2}{|c|}{ Community-onset BSIs } & \multicolumn{2}{|c|}{ Hospital-onset BSIs } & \multirow[t]{2}{*}{$p$ value* } \\
\hline & $\mathbf{N}$ & $\%$ & $\mathbf{N}$ & $\%$ & \\
\hline \multicolumn{6}{|l|}{ Patient demographics } \\
\hline Median age, years (range) & 72.6 & $69.3-75.8$ & 64.4 & $61.3-67.4$ & 0.0004 \\
\hline Male gender & 61 & 59.2 & 76 & 63.9 & 0.478 \\
\hline \multicolumn{6}{|l|}{ Underlying disease } \\
\hline Biliary tract disease & 35 & 34.0 & 17 & 14.3 & 0.0005 \\
\hline Diabetes mellitus & 33 & 32.0 & 27 & 22.7 & 0.118 \\
\hline \multicolumn{6}{|l|}{ Malignancy } \\
\hline Hematologic & 5 & 4.9 & 19 & 16.0 & 0.008 \\
\hline Solid organ & 43 & 41.8 & 37 & 31.1 & 0.099 \\
\hline Other & 15 & 14.6 & 34 & 28.6 & 0.012 \\
\hline None & 5 & 4.9 & 0 & 0 & 0.015 \\
\hline \multicolumn{6}{|l|}{ Site of infection } \\
\hline Liver abscess & 10 & 9.7 & 0 & 0 & 0.0005 \\
\hline Biliary tract infection & 43 & 41.8 & 17 & 14.3 & $<0.0001$ \\
\hline Other intra-abdominal infection & 5 & 4.9 & 6 & 5.0 & 0.949 \\
\hline Urinary tract infection & 20 & 19.4 & 31 & 26.1 & 0.241 \\
\hline Pneumonia & 10 & 9.7 & 12 & 10.1 & 0.926 \\
\hline Febrile neutropenia & 3 & 2.9 & 22 & 18.5 & 0.0003 \\
\hline Other/unknown & 14 & 13.6 & 31 & 26.1 & 0.021 \\
\hline \multicolumn{6}{|l|}{ Antimicrobial treatment } \\
\hline Penicillins & 21 & 20.4 & 28 & 23.5 & 0.574 \\
\hline Cephalosporins & 44 & 42.7 & 37 & 31.1 & 0.073 \\
\hline Carbapenems & 36 & 35.0 & 49 & 41.2 & 0.341 \\
\hline Other antimicrobials & 0 & 0 & 3 & 2.5 & 0.105 \\
\hline None & 1 & 1.0 & 4 & 3.4 & 0.231 \\
\hline 30-day survival $(n=213)$ & 79 & 82.3 & 99 & 84.6 & 0.648 \\
\hline \multicolumn{6}{|l|}{ Bacterial phenotypes } \\
\hline \multicolumn{6}{|l|}{ Capsular serotype } \\
\hline K1 & 10 & 9.7 & 5 & 4.2 & 0.103 \\
\hline K2 & 14 & 13.6 & 14 & 11.8 & 0.683 \\
\hline Non-K1/K2 & 79 & 76.7 & 100 & 84.0 & 0.168 \\
\hline Hypermucoviscosity & 15 & 14.6 & 6 & 5.0 & 0.016 \\
\hline \multicolumn{6}{|l|}{ Capsule-related gene $(n=133)$} \\
\hline magA gene & $6 / 52$ & 11.5 & $5 / 81$ & 6.2 & 0.273 \\
\hline rmpA gene & $11 / 52$ & 21.2 & $8 / 81$ & 6.2 & 0.010 \\
\hline \multicolumn{6}{|l|}{ Antimicrobial resistance } \\
\hline ESBL-producer & 2 & 1.9 & 22 & 18.5 & $<0.001$ \\
\hline Carbapenem resistance & 0 & 0 & 1 & 0.8 & 0.351 \\
\hline
\end{tabular}

*Pearson's $x^{2}$-test

which contrast to cases without liver abscesses in which $53 \%$ of BSI episodes were community-onset. Thirty-day mortality was not significantly different between cases with and without liver abscesses (30\% v. 18\%). Absence of any underlying disease was more often identified in cases with liver abscesses than in cases without liver abscesses $(20 \%$ v. $3 \%)$. In terms of bacterial characteristics, K1 capsular serotype and hypermucoviscosity phenotype were more often observed in isolates from patients with liver abscesses than in isolates from patients without liver abscesses (50\% v. $6 \%$ and $50 \%$ v. $10 \%$, respectively).

Based on these observations, we performed multivariate analyses to identify risk factors for liver abscess formation (Table 3). When adjusted by age, 
Table 2 Factors affecting the liver abscess formation

\begin{tabular}{|c|c|c|c|c|}
\hline Characteristics $(\mathrm{N}=127)$ & Liver abscess $(+)(\mathrm{N}=10)$ & Liver abscess $(-)(\mathrm{N}=117)$ & OR, 95\% Cl & p value* \\
\hline \multicolumn{5}{|l|}{ Patient demographics } \\
\hline Male gender & $7(70)$ & $73(62)$ & $1.41,0.35-5.72$ & 0.633 \\
\hline Age $\geq 65$ years & $8(80)$ & $86(74)$ & $1.44,0.29-7.16$ & 0.653 \\
\hline Community-onset BSIs & $10(100)$ & $62(53)$ & N/A & 0.004 \\
\hline \multicolumn{5}{|l|}{ Underlying disease } \\
\hline Biliary tract disease & $2(20)$ & $29(25)$ & $0.76,0.15-3.78$ & 0.735 \\
\hline Diabetes mellitus & $3(30)$ & $35(30)$ & $1.00,0.25-4.11$ & 0.996 \\
\hline \multicolumn{5}{|l|}{ Malignancy } \\
\hline Hematologic & $0(0)$ & $10(9)$ & 0 & 0.335 \\
\hline Solid organ & $2(20)$ & $33(28)$ & $0.64,0.13-3.15$ & 0.577 \\
\hline Other & $2(20)$ & $29(25)$ & $0.76,0.15-3.78$ & 0.735 \\
\hline None & $2(20)$ & $3(3)$ & $9.5,1.38-65.28$ & 0.007 \\
\hline 30-day mortality $(n=120)$ & $3(30)$ & $20(18)$ & $0.68,0.17-2.79$ & 0.363 \\
\hline \multicolumn{5}{|l|}{ Bacterial phenotypes } \\
\hline Capsular serotype K1 & $5(50)$ & $7(6)$ & $15.71,3.66-67.40$ & $<0.0001$ \\
\hline Capsular serotype K2 & $0(0)$ & $18(16)$ & 0 & 0.181 \\
\hline Hypermucoviscosity & $5(50)$ & $12(10)$ & $8.75,2.21-34.64$ & 0.0004 \\
\hline ESBL-producer & $0(0)$ & $8(7)$ & 0 & 0.393 \\
\hline
\end{tabular}

*Pearson's $X^{2}$-test

Table 3 Risk factors for liver abscess formation

\begin{tabular}{llll}
\hline Factors & aOR, 95\% Cl & p value $^{\mathbf{t}}$ & Points \\
\hline Community-onset BSIs & N/A & 0.0005 & 1 \\
Capsular serotype K1 & $26.47,4.56-206.38$ & 0.0003 & 1 \\
Hypermucoviscosity phenotype & $9.59,1.77-51.20$ & 0.010 & 1 \\
\hline
\end{tabular}

${ }^{\dagger}$ Pearson's $X^{2}$-test

a Adjusted by age, sex, and underlying diseases

sex, and underlying diseases, community-onset BSIs $(\mathrm{p}=0.0005), K 1$ capsular serotype $(\mathrm{p}=0.0003)$, and hypermucoviscosity phenotype $(\mathrm{p}=0.01)$ were identified as independent risk factors for liver abscess formation. Next, we evaluated whether these risk factors could be used as scores to identify cases with liver abscess formation. Each risk factor was given one point, so that the total scores would be from zero to three points (Table 3). To determine the cut-off value for the identification of cases with liver abscesses, we performed receiver operating characteristic (ROC) curve analysis (Fig. 1). The area under ROC was 0.90, indicating that the analysis was highly accurate. The sensitivity and specificity for each score were shown in Table 4 . The score of $\geq 2$ points provided maximal Youden's index with a sensitivity and specificity of 0.70 and 0.94 , respectively.

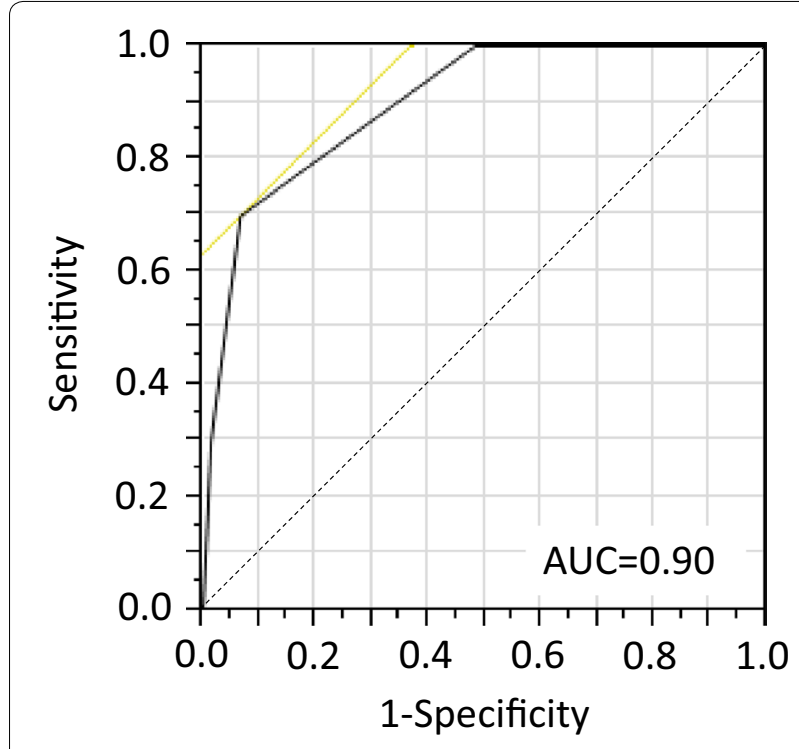

Fig. 1 Receiver operator characteristic curve analysis for the scoring system

\section{Discussion}

The definition of HVKp is not formally defined. The reason may be that the precise meaning of the term "hypervirulent" in this context is ambiguous. In our study, we determined the definition of HVKp infections as "infections by $K$. pneumoniae complicated by liver abscess formation" with the assumption that CKp 
Table 4 Accuracy of the proposed scoring system for the diagnosis of HV K. pneumoniae infection

\begin{tabular}{llllll}
\hline Score & $\begin{array}{l}\text { Liver } \\
\text { abscess } \\
(+)\end{array}$ & $\begin{array}{l}\text { Liver } \\
\text { abscess } \\
(-)\end{array}$ & Sensitivity & Specificity & $\begin{array}{l}\text { Youden's } \\
\text { index }(\boldsymbol{J})\end{array}$ \\
\hline$\geq 0$ & 10 & 212 & 1 & 0 & 0 \\
$\geq 1$ & 10 & 101 & 1 & 0.52 & 0.52 \\
$\geq 2$ & 7 & 12 & 0.70 & 0.94 & 0.64 \\
3 & 3 & 3 & 0.30 & 0.99 & 0.29 \\
$\begin{array}{l}\text { Number in } \\
\text { sample }\end{array}$ & 10 & 212 & & & \\
\hline
\end{tabular}

infections could never lead to liver abscess formation. This definition may contradict to literatures in which bacterial phenomenon of hypermucoviscosity was used as the definition for HVKp infections. Our idea is in line with the review by Catalán-Nájera et al., which suggested that the hypermucoviscosity and the hypervirulence are two different phenotypes that should not be used synonymously [16].

One of the most notorious characteristics of HVKp infections is its tendency to cause metastatic infections such as endophthalmitis. The treatment of bacterial endophthalmitis is difficult, and total loss of vision may ensue in the affected eyes [17]. Furthermore, even if metastatic infections are not obvious in early stage of infections, they may become apparent in later stage of infections as was noticed in our case. Thus, to recognize the possibility of these complications, it should be important to promptly identify cases with the risk for these complications. For this purpose, identification of patients at-risk of liver abscess formation should be sufficient, since metastatic infections are accompanied by liver abscess formation in HVKp infections.

Through the analyses of the patient cohort of $K$. pneumoniae BSIs, we identified three factors, namely, community-onset BSIs, the hypermucoviscosity phenotype, and capsular serotype $\mathrm{K} 1$ of the isolated strains, as risk factors for liver abscess formation. By utilizing these risk factors as points, we developed a scoring system. The system seems to be accurate with the AUROC of 0.90, and the maximal Youden's index was obtained when the score was $\geq 2$ points. This scoring system might be a useful tool to identify patients at-risk of HVKp infections, and prospective evaluation of this system should be performed.

Among the points for the scoring system, the identification of community-onset infection and the hypermucoviscosity phenotype should be a straightforward process. The maneuver of the string test is simple and should be available to almost all clinical microbiology laboratories. Compared to these factors, identification of $K$. pneumoniae capsular serotype is not an easy task. Quellung method, which is the traditional method for the identification of $K$. pneumoniae capsular serotype [18], is laborious and outdated. Other method using antisera [19] could be done in reference laboratories or research institutions but would be difficult to perform as routine practices in most laboratories. PCR method is usually employed to determine the capsular serotype but is laborious and not logistically applicable to all hospital settings. Compared to these methods, the immunochromatographic method [20] is very simple to perform and is possible to obtain the result within a few minutes. Even though the commercial product of this method is currently approved only in Taiwan for the clinical diagnosis of HVKp infections, and it can detect only capsular serotypes $\mathrm{K} 1$ and $\mathrm{K} 2$, it is an attractive tool to quickly identify capsular serotype K1. When this method is available, by using isolated colonies the clinical microbiologists can detect both the hypermucoviscosity phenotype and capsular serotype K1 or K2 within a short time and inform the clinicians promptly. In our experience, the whole process of calculating the scores, including the chart review and microbiology analyses, and providing any recommendation regarding the possible complications associated with HVKp infections could be completed within $1 \mathrm{~h}$.

Our study has some notable limitations. First, the study was performed in a single-center setting, so that some important risk factors for HVKp infections may be missed. In addition, the fact that all patients except one were of Japanese ethnicity may have biased the types of risk factors in an ethnicity-related way. Second, not all patients with $K$. pneumoniae BSIs received CT scans, so that some cases of liver abscess may be missing. In this study the decision to perform CT scans was at the discretion of attending physicians. However, through the investigation of patient charts who did not receive $C T$ scans, the risk of the development of liver abscesses appeared to be minimal in these patients, and we assume that all cases of liver abscess could be identified in our study. Third, we did not pick up the genetic characteristics of isolates as risk factors for HV infections. $R m p A$ gene was preferentially detected in isolates from community-onset BSIs, and it could be an additional risk factor for $\mathrm{HV}$ infection. However, since the purpose of this study was to develop simple scoring system which can be performed in most microbiology laboratories as routine practices, the exclusion of genetic analyses should be justified. 


\section{Conclusions}

We analyzed the patient cohort of $K$. pneumoniae BSIs and identified three factors, community-onset infection, hypermucoviscosity phenotype, and capsular serotype $\mathrm{K} 1$, as risk factors for $\mathrm{HVKp}$ infections. Based on the results we developed a simple scoring system to identify HVKp infections with the sensitivity of $70 \%$ and the specificity of $94 \%$ when scores are $\geq 2$ points. This scoring system may enable every clinician to efficiently identify patients with $K$. pneumoniae BSIs who are at risk for HVKp infections and its associated metastatic complications.

\section{Methods}

\section{Study design and case definition}

The study was conducted in a 900-bed tertiary-care university hospital from April 2009 to May 2019. Episodes of $K$. pneumoniae BSIs were identified from the records of the clinical microbiology laboratory. If multiple episodes of $K$. pneumoniae BSIs were identified in a patient, only the first episode was involved in the study. All patients except one were of Japanese ethnicity. Clinical characteristics were collected from the electronic health record of each patient. K. pneumoniae BSIs identified $\leq 72 \mathrm{~h}$ or $>72 \mathrm{~h}$ after the admission to the hospital were designated as community-onset or hospital-onset infections, respectively. Hypervirulent K. pneumoniae infections was defined as infections by $K$. pneumoniae associated with liver abscess formation. The study was approved by the institutional review board (No. 18-11-03).

\section{Microbiology}

Blood isolates were identified as K. pneumoniae by Vitek II system (bioMerieux, Japan) and/or MALDI TOF-MS (Bruker, Japan). Isolates were frozen at $-80{ }^{\circ} \mathrm{C}$ until analysis. Isolates were cultured on Columbia agar with $5 \%$ sheep blood (Nippon Becton-Dickinson, Japan) overnight. String tests were performed on all isolates. The string test was diagnosed as positive when a bacterial inoculation loop produced a viscous string of $>5 \mathrm{~mm}$ length by stretching bacterial colonies on agar plates. $K$. pneumoniae strains with a positive string test were determined as having the hypermucoviscosity phenotype.

\section{Capsular serotype analysis}

For isolates from 2009 to 2016, presence of capsular polysaccharide $\mathrm{K} 1$ and $\mathrm{K} 2$ genes was determined by polymerase chain reaction (PCR) as described previously [10]. In 2017, PCR method was switched to immunochromatography method by utilizing the rapid testing cassette for the detection of capsular serotypes K1 and K2 (KeMyth Biotech Co., Taiwan) [20]. By using the rapid testing cassettes, it is possible to identify the capsular serotypes as $\mathrm{K} 1, \mathrm{~K} 2$, or non- $\mathrm{K} 1 / \mathrm{K} 2$ within several minutes. Before switching the method, we evaluated the performance of the rapid testing cassettes by comparing the results of PCR with the rapid testing cassette to identify capsular serotype $\mathrm{K} 1$ and $\mathrm{K} 2$. Analysis of 23 isolates showed that the test results by rapid testing cassettes matched completely with the test results by PCR (unpublished data).

\section{Statistics}

Patient demographics and laboratory data were processed by JMP software ver. 10 (SAS Institute Japan, Tokyo, Japan). The $\chi^{2}$ test was used to compare categorical variables. Student's t-test was used to compare numerical variables. Logistic regression analyses were used to determine which risk factors were statistically significant for each category. Receiver operating characteristic curve was used to determine the cut-off value for the assessment of $\mathrm{HV} K$. pneumoniae infections based on the scoring of each risk factor.

\section{Acknowledgements \\ We thank technicians in the clinical microbiology laboratory of Fukuoka University Hospital for their work in microbiological analyses.}

\section{Authors' contributions}

Conceptualization, AT; Methodology, AT and MY; Investigation, AT, MY and CT; Writing-original draft, AT; Writing-review and editing, MY, CT, AM, TT, and $Y T$; Supervision, AT; Funding acquisition, AT and $Y \mathrm{~T}$. All authors read and approved the final manuscript.

Funding

This study was funded by institutional internal funding.

Availability of data and materials

The datasets used and/or analysed during the current study are available from the corresponding author on reasonable request.

\section{Ethics approval and consent to participate}

This study was approved by the institutional review board of Fukuoka University Hospital (No. 18-11-03). Consent to participate in this study from each patient was waived by the review board due to the retrospective nature of this study.

Consent for publication

Not applicable.

Competing interests

The authors declare that they have no competing interests.

\footnotetext{
Author details

${ }^{1}$ Department of Medical Oncology, Hematology, and Infectious Diseases, Fukuoka University Hospital, 7-45-1 Nanakuma, Jonan, Fukuoka 8140180, Japan. ${ }^{2}$ Department of Infection Prevention and Control, Fukuoka University Hospital, 7-45-1 Nanakuma, Jonan, Fukuoka 8140180, Japan. ${ }^{3}$ Department of Laboratory Medicine, Fukuoka University Hospital, 7-45-1 Nanakuma, Jonan, Fukuoka 8140180, Japan. ${ }^{4}$ Present Address: Department of Developmental Medicine, Research Institute, Osaka Women's and Children's Hospital, Osaka, Japan.
} 
Received: 9 May 2020 Accepted: 6 July 2020

Published online: 09 July 2020

\section{References}

1. Podschun R, Ullmann U. Klebsiella spp. as nosocomial pathogen: epidemiology, taxonomy, typing methods, and pathogenicity factors. Clin Microbiol Rev. 1998;11:589-603.

2. Liu YC, Cheng DL, Lin CL. Klebsiella pneumoniae liver abscess associated with septic endophthalmitis. Arch Intern Med. 1986;146:1913-6.

3. Casanova C, Lorente JA, Carrillo F, Pérez-Rodríguez E, Núñez N. Klebsiella pneumoniae liver abscess associated with septic endophthalmitis. Arch Intern Med. 1989;149:1467.

4. Saccente M. Klebsiella pneumoniae liver abscess, endophthalmitis, and meningitis in a man with newly recognized diabetes mellitus. Clin Infect Dis. 1999:29:1570-1

5. Hu BS, Lau YJ, Shi ZY, Lin YH. Necrotizing fasciitis associated with Klebsiella pneumoniae liver abscess. Clin Infect Dis. 1999;29:1360-1.

6. Siu LK, Yeh KM, Lin JC, Fung CP, Chang FY. Klebsiella pneumoniae liver abscess: a new invasive syndrome. Lancet Infect Dis. 2012;12:881-7.

7. Shon AS, Bajwa RP, Russo TA. Hypervirulent (hypermucoviscous) Klebsiella pneumoniae: a new and dangerous breed. Virulence. 2013;4:107-18.

8. Paczosa MK, Mecsas J. Klebsiella pneumoniae: going on the offense with a strong defense. Microbiol Mol Biol Rev. 2016;80:629-61.

9. Fang CT, Chuang YP, Shun CT, Chang SC, Wang JT. A novel virulence gene in Klebsiella pneumoniae strains causing primary liver abscess and septic metastatic complications. J Exp Med. 2004;199:697-705.

10. Fang CT, Lai SY, Yi WC, Hsueh PR, Liu KL, Chang SC. Klebsiella pneumoniae genotype K1: an emerging pathogen that causes septic ocular or central nervous system complications from pyogenic liver abscess. Clin Infect Dis. 2007;45:284-93.

11. Li J, Ren J, Wang W, Wang G, Gu G, Wu X, et al. Risk factors and clinical outcomes of hypervirulent Klebsiella pneumoniae induced bloodstream infections. Eur J Clin Microbiol Infect Dis. 2018:37:679-89.
12. Harada S, Aoki K, Yamamoto S, Ishii Y, Sekiya N, Kurai H, et al. Clinical and molecular characteristics of Klebsiella pneumoniae isolates causing bloodstream infections in Japan: occurrence of hypervirulent infections in health care. J Clin Microbiol. 2019;57:e01206-19.

13. Yu WL, Ko WC, Cheng KC, Lee HC, Ke DS, Lee CC, et al. Association between rmpA and magA genes and clinical syndromes caused by Klebsiella pneumoniae in Taiwan. Clin Infect Dis. 2006:42(10):1351-8.

14. Alsaif HS, Venkatesh SK, Chan DS, Archuleta S. CT appearance of pyogenic liver abscesses caused by Klebsiella pneumoniae. Radiology. 2011;260:129-38

15. Lee NK, Kim S, Lee JW, Jeong YJ, Lee SH, Heo J, Kang DH. CT differentiation of pyogenic liver abscesses caused by Klebsiella pneumoniae vs non-Klebsiella pneumoniae. Br J Radiol. 2011;84:518-25.

16. Catalán-Nájera JC, Garza-Ramos U, Barrios-Camacho H. Hypervirulence and hypermucoviscosity: two different but complementary Klebsiella spp. phenotypes? Virulence. 2017;8:1111-23.

17. Durand ML. Bacterial and fungal endophthalmitis. Clin Microbiol Rev. 2017;30:597-613.

18. Riser E, Noone P, Poulton TA. A new serotyping method for Klebsiella species: development of the technique. J Clin Pathol. 1976;29:296-304.

19. Chuang YP, Fang CT, Lai SY, Chang SC, Wang JT. Genetic determinants of capsular serotype K1 of Klebsiella pneumoniae causing primary pyogenic liver abscess. J Infect Dis. 2006;193:645-54.

20. Siu LK, Tsai YK, Lin JC, Chen TL, Fung CP, Chang FY. Development of a colloidal gold-based immunochromatographic strip for rapid detection of Klebsiella pneumoniae serotypes K1 and K2. J Clin Microbiol. 2016:54:3018-21.

\section{Publisher's Note}

Springer Nature remains neutral with regard to jurisdictional claims in published maps and institutional affiliations.
Ready to submit your research? Choose BMC and benefit from:

- fast, convenient online submission

- thorough peer review by experienced researchers in your field

- rapid publication on acceptance

- support for research data, including large and complex data types

- gold Open Access which fosters wider collaboration and increased citations

- maximum visibility for your research: over $100 \mathrm{M}$ website views per year

At $\mathrm{BMC}$, research is always in progress.

Learn more biomedcentral.com/submissions 